\title{
mTOR Signaling in Regulatory T Cell Differentiation and Expansion
}

\author{
Cristin Coquillard, Valery Vilchez, Francesc Marti, Roberto Gedaly* \\ Department of Surgery, Division of Transplantation, University of Kentucky - College of Medicine, 800, Rose Street, C453 Lexington, KY, 40536, USA
}

\begin{abstract}
Received: March 12, 2015; Accepted: March 30, 2015; Published: April 15, 2015
*Corresponding author: Roberto Gedaly, Department of Surgery, Division of Transplantation, University of Kentucky, College of Medicine, 800, Rose Street, C453 Lexington, KY, 40536, USA, Tel: + 859-323-4661; Fax: + 859-257-3644; E-mail: rgeda2@uky.edu
\end{abstract}

\begin{abstract}
mTOR signaling is gaining recognition for its role in dictating $\mathrm{T}$ cell fate through the interaction and balance between two complexes, mTORC1 and mTORC2. naive T cells do not require mTOR for initial T Cell Receptor (TCR)-mediated cytokine production. However, mTOR plays a critical role in determining antigen-induced effector and regulatory $\mathrm{T}$ cell (Treg) fate decisions. mTOR activation is essential for T cell commitment to Th1, Th2 and Th17 effector lineages. In the absence of mTOR, naive T cells preferentially differentiate into Treg cells. Previous studies have placed mTOR as a critical mediator of Treg development and function, suggesting that specific targeting could lead to new therapeutic opportunities in generating tolerance. This review focuses on the recent advances in mTOR signaling and downstream events in $\mathrm{T}$ cells, emphasizing the contrasting role of mTOR in effector versus Treg cells and its clinical implications.
\end{abstract}

Keywords: Regulatory T cells; mTOR signaling; mTOR inhibition; Rapamycin

\section{Abbreviations}

APC: Antigen Presenting Cells; AMPK: AMP-Activated Protein Kinase; CTLA4: Cytotoxic T Lymphocyte Antigen 4; eIF4E: Eukaryotic Translation Initiation Factor 4E; FKBP12: FK506-Binding Protein; FRB: FKBP12-Rapamycin Binding; F0X01: Forkhead Box 01; FOX03: Forkhead Box 03; GITR: Glucocorticoid-Induced Necrosis Factor Receptor; IL-2: Interleukin 2; IL-2R: Interleukein-2 Receptor; mTOR: Mammalian Target of Rapamycin; mTORC1: Mammalian Target of Rapamycin Complex 1; mTORC2: Mammalian Target of Rapamycin Complex 2; PI3K: Phosphatidylinositol-3-OH Kinase; PPAR-g: Peroxisome Proliferator-Activated Receptor-Gamma; PRAS40: Proline-Rich AKT/ PKB Substrate; PTEN: phosphatase and Tensin Homolog; Raptor: Regulatory Protein associated with mTOR; Rictor: Rapamycin-Insensitive Companion of mTOR; SGK1: Serum Glucocorticoid-Regulated Kinase 1; S6K: p70 Ribosomal S6 Kinase; SREBP1: Sterol Regulatory Binding Protein 1; TCR: T Cell Receptor; TSC1: Tuberous Sclerosis Complex 1; TSC2: Tuberous Sclerosis Complex 2; ULK1: UNC-51-like Kinase

\section{Introduction}

Appropriate T cell Activation Requires Three Major Signals:
T Cell Receptor (TCR) engagement, co-stimulatory signals, and the presence of inflammatory cytokines. These signals are sensed by mTOR. Once activated, mTOR interacts with and influences T cell signaling pathways essential in regulating cell differentiation, proliferation, survival and metabolism. Multiple agonists (TCR engagement and co-stimulation, growths factors, and cytokines) regulate $\mathrm{T}$ cell fate by acting on cell surface plasma membrane receptors and stimulating mTOR through the activation of PI3KAKT and the Ras-ERK-RSK signaling pathways [1].

The mTOR signaling pathway plays a crucial role in dictating $\mathrm{T}$ cell fate through the interaction and balance of two mTORcontaining complexes, mTOR Complex 1 (mTORC1) and mTOR Complex 2 (mTORC2). mTORC1 and mTORC2 are involved in a distinct set of cellular signals and cause separate downstream effects $[2,3]$.

PI3K/mTOR signaling has been shown to promote effector $\mathrm{T}$ cell activation and differentiation, and our group and others have demonstrated the reorganization of this pathway during the differentiation of naive $\mathrm{T}$ cells into functional suppressor Tregs $[4,5]$. Indeed, $\mathrm{T}$ cell specific loss of mTOR correlates with a diminished generation of effector Th1, Th2 and Th17 cells, and enhanced generation of Tregs. Tregs play a major role in the prevention of autoimmunity by suppressing $\mathrm{T}$ cell responses to self-antigens and by limiting the response to foreign antigens. These cells consistently express high levels of the cell surface IL-2 Receptor (IL-2R) $\alpha$-chain (CD25) and the forkhead family transcription regulator, Foxp3. Rapamycin and its analogs (rapalogs) promote tolerance in experimental models by favoring Treg-dependent suppression in human T cells, and are currently being used to prevent rejection in solid organ transplantation $[6,7]$.

As mTOR is emerging as a critical regulator of the balance between regulatory and effector $\mathrm{T}$ cells, there is increasing interest in the development of molecular-targeted therapies that control upstream and downstream events of mTOR activities. The focus of our manuscript is to describe the contrasting role of mTOR in effector versus Treg cells and the clinical implications of its inhibition. 


\section{mTOR structure and complexes}

The TORs are large proteins $(\sim 250 \mathrm{kDa})$ belonging to the phosphatidylinositol kinase-related kinase family (PIKK), which are characterized by a carboxy-terminal serine/ threonine kinase domain similar in sequence to that found in the phosphatidylinositol 3-kinases [8]. At its amino-terminal, mTOR contains 20 HEAT (Huntington, EF3, A subunit of PP2A, TOR1) repeats, which function as protein interaction sites, followed by a large central FAT (FRAP, ATM, TRAP) domain. Toward the carboxy-terminal, mTOR includes a FRB (FKB12-rapamycin binding) domain, a kinase domain and a second FAT domain called the FATC domain (FAT C-terminus) [9] [Figure 1A].

In mammalian cells, mTOR exists as the catalytic subunit of two structurally and functionally different complexes, mTORC1 and mTORC2. Each is distinguished by a unique protein essential for assembly of the complex, binding substrate, and regulation [2]. In mTORC1, that protein is Raptor (regulatory protein associated with mTOR) while in mTORC2 it is Rictor (Rapamycin-insensitive companion of mTOR) $[3,10]$. Other components are also unique to each complex: mTORC1 contains PRAS40 (Proline-rich AKT/ PKB substrate $40 \mathrm{kDa}$ ), which acts as a negative regulator of mTOR [11] while mTORC2 contains Protor and mSIN1, both of which appear to play a role in downstream events [12,15]. In addition to these unique proteins, both complexes share mLST8 and Deptor [2] [Figure 1B].

\section{mTOR signaling}

mTORC1 integrates information from multiple pathways, including growth factors, nutrients, oxygen, and energy status. The interplay of these complex signals converges to promote cell growth and proliferation by increasing anabolic processes like protein and lipid synthesis and decreasing catabolic processes like autophagy [14]. Much of what is known about mTORC1 arose from studies using rapamycin, which binds to the FRB domain of mTOR along with FKBP12 (FK506-binding protein of $12 \mathrm{kDa}$ ) and inhibits its function[15]. mTORC1 is thus known as the rapamycin-sensitive complex. Compared to mTORC1, relatively little is known about the regulation of mTORC2. To categorize mTORC2 as a rapamycin-insensitive complex is a slight misnomer due to rapamycin's ability to inhibit mTORC2 at high dose and/or after prolonged use [16]. When activated, mTORC2 phosphorylates the kinase AKT at Serine 473 and induces its full activation [17]. Since AKT is also upstream activator of mTORC1 [18], AKT activation has an important role in mTORC1-mTORC2 crosstalk as one of the multiple intertwined bioloops that regulate mTOR activity. The general consensus suggests that mTORC2 is activated by growth factors, and that this complex plays a role in rearrangement of the cytoskeleton and in cell cycle progression,

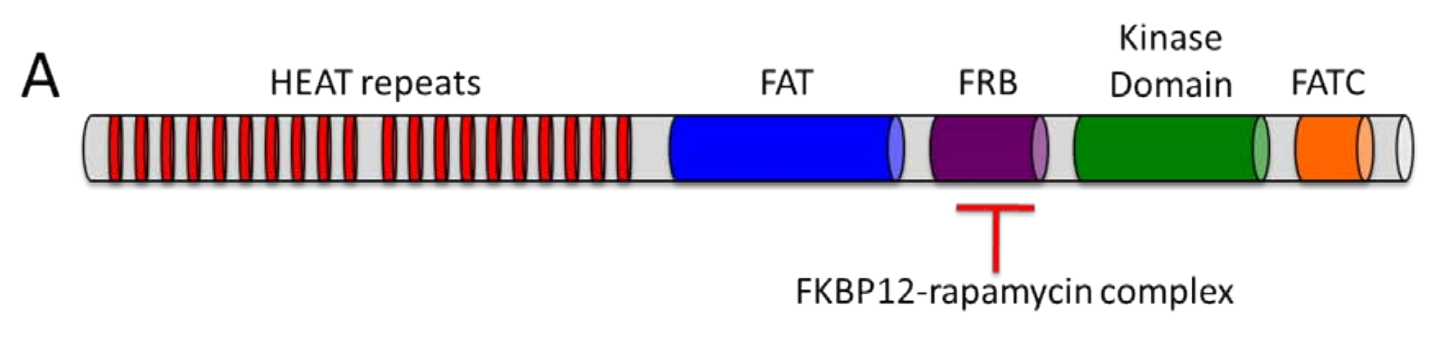

B

mTOR complex 1 (mTORC1)

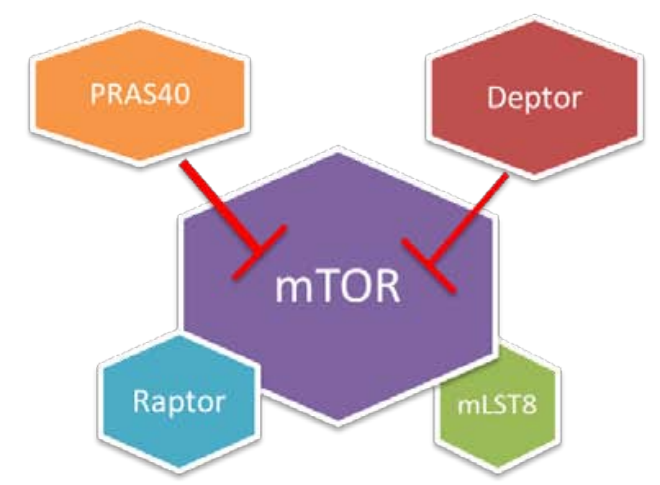

mTOR complex 2 (mTORC2)

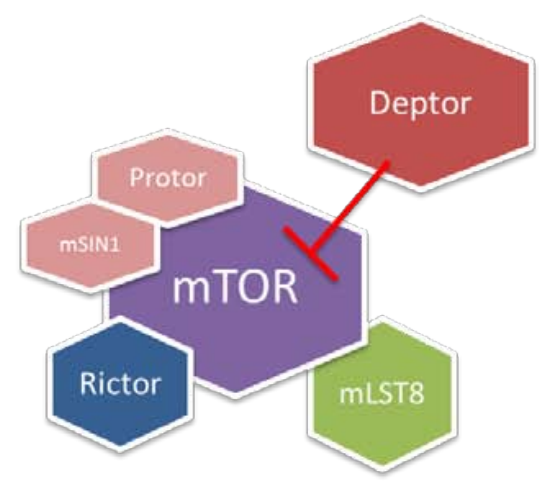

Figure 1: The primary structure of mTOR including HEAT repeats, FAT, FRB, Kinase, and FATC domains. B. The protein subunits of the mTOR complexes, both complexes include, in addition to mTOR, mLST8 and Deptor, which acts as an inhibitor of both complexes. mTORC1 additionally includes PRAS40, an inhibitor of complex 1, and Raptor. mTORC2 also includes Rictor, Protor, and mSIN1. 


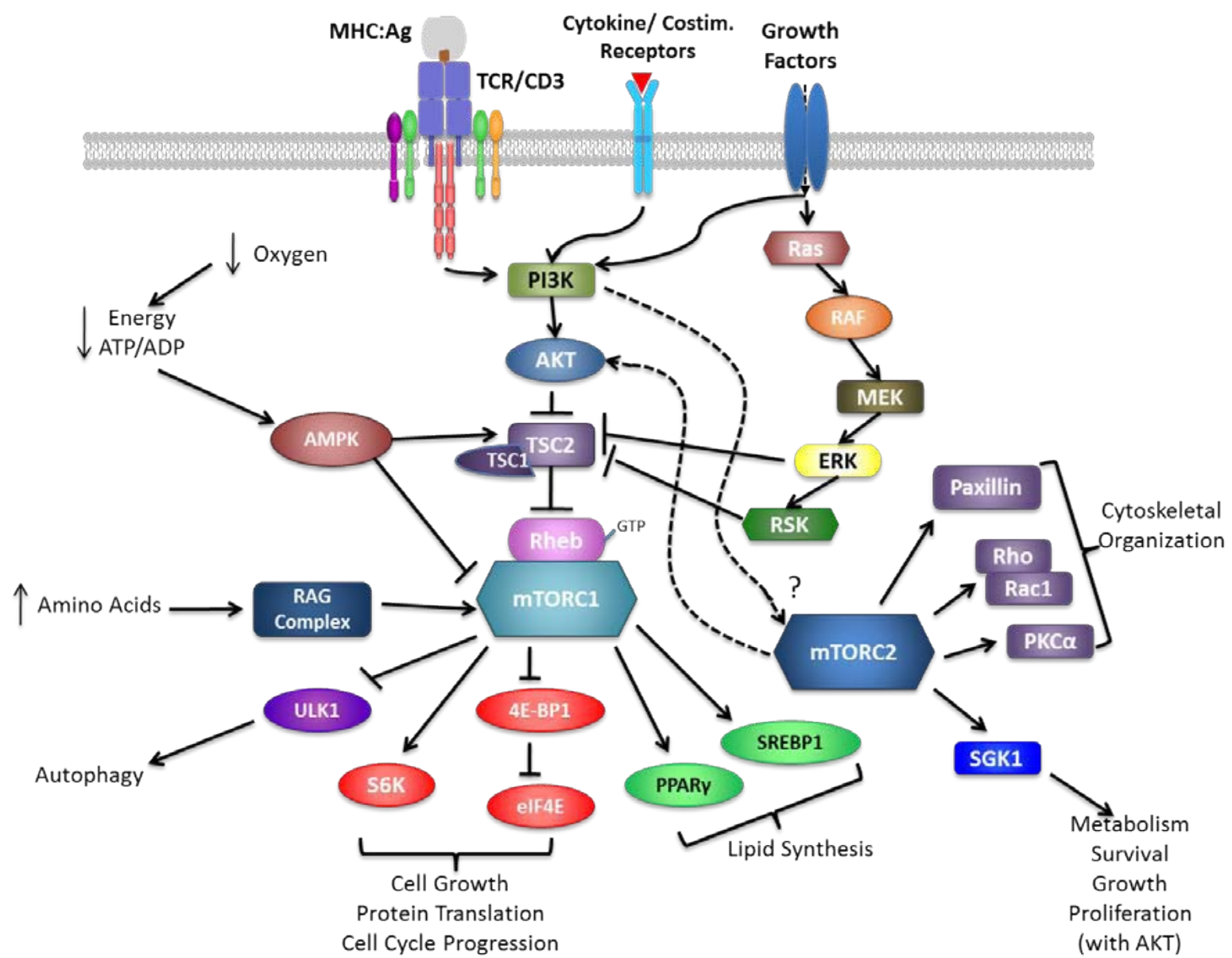

Figure 2: Simplified depiction of the mTOR signaling pathway. m mTOR complex 1 (mTORC1) is activated by extracellular signals like TCR engagement and CD28 co stimulation, insulin, and growth factors via the PI3K/ Akt and Ras/ RAF/ MED/ ERK/ RSK pathways. Intracellularly, sufficient amino acids necessary for mTORC1 activation where as low oxygen and energy inhibit mTORC1. Downstream, mTORC1 activates cell growth and protein translation through S6K and eIF4E as well as lipid synthesis through PPAR-gamma and SREBP1, while inhibiting autophagy through ULK1. Other signaling pathways also affect mTORC1 that are not depicted here, including Wnt and TNF-alpha. The signaling pathways are not as well elucidated for mTOR complex 2 (mTORC2). It is thought to be activated by growth factors through a PI3K pathway that differs from that of mTORC1. Downstream, mTORC2 activates Akt, putting it upstream of mTORC1 signaling, and SGK1, both of which influence metabolism, survival, growth, and proliferation. mTORC2 also influences cytoskeletal organization through activation of Paxillin, PKC- alpha, Rho, and Rac1

cell survival, and anabolism by phosphorylating the ACG kinases in a pathway that intersects with the activation of mTORC1 [2]. An overview of the mTOR complexes and signaling pathway is summarized in Figure 2.

\section{The Yin-Yang of mTORC1 and mTORC2}

\section{Upstream and downstream events of mTORC1}

The tuberous sclerosis complex, a heterodimer comprised of TSC1 and TSC2, is the point at which signals from several different cellular pathways are integrated in the regulation of mTORC1. TSC1/2 acts as a GTPase-activating protein (GAP) towards Rheb, promoting the hydrolysis of Rheb bound to GTP, and converting it to an inactive, GDP bound state. TSC1/2 therefore functions as a negative regulator of mTORC1 $[19,20]$. When Rheb is in an active GTP bound state, its translocation to the cell membrane stimulates mTORC1-mediated signaling [19].
Regulation of the TSC complex is primarily mediated by two major signaling pathways: the PI3K-AKT and the RAS-ERK axis. Binding of insulin or other growth factors or cytokines to their cell-surface receptors activates PI3K, which results in the activation of AKT. AKT then directly phosphorylates TSC2 on several residues, there by inhibiting its GAP activity for Rheb and allowing activation of mTORC1 [21]. AKT also directly phosphorylates PRAS40, relieving its inhibition of mTORC1 [22]. The tumor suppressor PTEN (phosphatase and tensin homolog deleted on chromosome 10) opposes the activity of PI3K and negatively regulates this pathway [22]. Alternatively, growth factor-induced RAS-ERK pathway can activate mTORC1 through ERK and RSK-mediated phosphorylation of TSC2 and/or Raptor [23]. Other pathways including TNF and Wnt/ $\beta$-catenin signaling have also been described as extracellular upstream regulators of mTORC1 [24]. Likewise, AMPK signaling inhibits mTORC1 when 
intracellular conditions like hypoxia and low cellular energy are detected $[14,25,26]$.

Activated mTORC1 acts on a broad range of downstream substrates [15]. One mechanism by which mTORC1 induces protein synthesis is through inhibition of the eukaryotic initiation factor 4E binding protein 1 (4E-BP1), which itself acts as a translation inhibitor [27]. Another major downstream target of mTORC1 is the p70 ribosomal S6 kinase (S6K). When activated, S6K upregulates protein translation and elongation, mRNA synthesis, and ribosome biogenesis [2,27]. In a regulatory feedback loop, S6K also acts upstream of mTORC1 by inhibiting PI3K activation and rebalancing mTOR activity $[15,28,29]$. In this context, the preferential short-term inhibition of mTORC1 by rapamycin and the subsequent loss of the S6K negative feedback loop may lead to unexpected secondary effects such as hyperactivation of AKT and induction of mTORC2 downstream effects.

\section{Upstream and downstream of mTORC2}

The activation of mTORC2 is significantly less well understood than that of mTORC1. Early studies demonstrated that mTORC2 was activated by insulin via the PI3K pathway, but signaling steps beyond PI3K are distinct from mTORC1 activation and have mostly remained unclear. Recent studies have implicated ribosomal binding of $\mathrm{mTORC2}$ and ER stress in mTORC2 signaling, but further studies are needed to fully understand the signaling inputs to mTORC2 [30,31].

Similarly, not much is known regarding the downstream effects of mTORC2 and its role in the regulation of cellular functions. mTORC2 controls the actin cytoskeleton by promoting the activation of the ACG kinase protein kinase C-alpha (PKC $\alpha$ ), inducing GTP loading of the GTPases Rho and Rac1, and the phosphorylation and relocalization of paxillin [10]. Along with PKC $\alpha$, mTORC2 also activates two other members of the AGC family of kinases-SGK1 and AKT. Some of the main downstream effects of mTORC2 are caused by its inhibition of the transcription repressors FOX01 and FOX03 (Forkhead box 01/03).

mTORC1 and mTORC2 activities are affected by crossregulatory loops promoted by downstream events of both complexes. As previously mentioned, one important mechanism of cross-talk involves mTOR regulation of AKT. mTORC2 phosphorylates AKT at serine 473, which, together with the phosphorylation at threonine 308 (PI3K-dependent activation of PDK1), is needed for complete AKT activity [17]. Physiological or pharmacological inhibition of mTORC1 (by Deptor in cancer cells or by rapamycin) may indirectly promote the activation of AKT by relieving the negative feedback of S6K on PI3K [32]. The complexities of the mTORC1 and mTORC2 signaling and crosstalk have been excellently and thoroughly reviewed in recent publications $[2,15,33,34]$.

\section{mTOR signaling on $\mathrm{T}$ cell fate decisions}

Naive mature $\mathrm{T}$ cells circulate in the periphery in a quiescent state characterized by small size and exit from active division (G0). Regular engagement of the TCR with self-peptide presented by MHC, and the antiapoptotic interaction of IL-7 with IL-7R, promote survival of these cells in this resting state [35]. In quiescent $\mathrm{T}$ cells, homeostasis and the initial TCR-mediated cytokine production are surprisingly independent of mTOR signaling [36]. Deficiency of TSC1 is highly disruptive of naive $\mathrm{T}$ cell homeostasis, which indicates the important role of TSC1 and the requirement of low functional mTORC1 during $\mathrm{T}$ cell quiescence [37,38].

Recognition of foreign antigen is necessary to activate effector T cells, but engagement of the TCR with the antigen: MHC complex alone (signal 1) is not sufficient for activation. Rather, co-stimulation with a second signal (signal 2) like CD28 is required for full activation, which induces the production of IL-2 necessary for $\mathrm{T}$ cell activation and proliferation (signal 3) [31]. In the absence of signal 2, signal 1 alone induces a $\mathrm{T}$ cell anergic state, characterized by hypo responsiveness to secondary TCR stimulation [39]. Anergy is likely among the most prominent mechanisms in controlling peripheral tolerance to self-antigen. Engagement of the TCR can induce low-level activation of mTOR through the PI3K pathway, but this is amplified by signal 2, which strongly activates the PI3K/ AKT/ mTOR axis [40].

Previous studies indicated that mTOR inhibition with rapamycin induces $\mathrm{T}$ cell anergy even in the presence of costimulation, thus highlighting the importance of mTOR signaling in effective $\mathrm{T}$ cell activation [35]. In contrast, recent studies support that the absence of mTOR does not alter the TCR-induced signaling cascade and the ability of naive T cells to produce IL- 2 . The same naive $\mathrm{T}$ cells fail to differentiate into an effector cells under skewing conditions both in vitro and in vivo [37].

\section{The role of $\mathrm{mTOR}$ in effector $\mathrm{T}$ cells}

The activation status of the cell and the integration of multiple signals from the local environment determine the intensity, duration and type of mTOR signaling [41]. During TCR activation, naive $\mathrm{CD} 4^{+} \mathrm{T}$ cells can differentiate into effector Th1, Th2, or Th17 cells or suppressor iTregs depending on the particular cytokine milieu where the activation occurs. An environment rich in IL-12 and IFN- $\gamma$ will likely produce Th1 cells, while the presence of IL-6, IL-21, and TGF- $\beta$ will skew differentiation towards the production of Th17cells [42]. Though quite different in their functions, Th1 and Th17 cells share a common intracellular signaling pathway; and the differentiation of each of them requires signaling via the mTORC1 complex [43]. In contrast, there are conflicting reports on the role of mTORC2 in Th1 differentiation. A study by Lee et al. [44] reported that naive $\mathrm{T}$ cells are unable to differentiate into the Th1 lineage when deficient in Rictor or Rheb, although later studies indicate otherwise [45]. There is consensus that Th2 cells fail to differentiate in the absence of Rictor but develop normally in the absence of Rheb, pointing to mTORC2 rather than mTORC1 as key regulator of Th2 differentiation [43, 44].

\section{The role of mTOR in treg cells}

Treg cells are critical in toning down the immune response to prevent a pathological inflammatory response or autoimmunity [46]. Though it became clear they existed decades ago, it was not until 2003 that the phenotype of suppressor Treg cells was characterized as $\mathrm{CD}^{+} \mathrm{CD} 25^{+} \mathrm{Foxp}^{+}$, although none of these 
markers are exclusively expressed in Tregs. Tregs express, among others, cytotoxic T lymphocyte antigen 4 (CTLA4) and glucocorticoid-induced necrosis factor receptor (GITR), but do not express the IL-7 receptor (CD127) [47]. Tregs comprise only a small percentage of the $\mathrm{T}$ cells in the body (5-10\%), but they are absolutely necessary for immune homeostasis.

Treg cells exist in two phenotypically indistinguishable yet distinct subtypes that differ in their origin: natural Tregs (nTregs) and induced Tregs (iTregs). nTregs develop in the thymus, mainly in response to self-antigens and require high affinity interactions between the TCR and peptide: MHC with CD28 co-stimulation. iTregs develop from naive CD4+CD25- T cells in the periphery [48], and their repertoire is more specific for foreign antigens [49]. In contrast to nTregs, iTregs have a lower activation threshold for TCR stimulation and have no requirement for CD28 co-stimulation, although they require IL- 2 and TGF- $\beta$ signals. The differences in antigen-recognition between nTregs (self-antigen) and iTregs (foreign) may partially account for their distinct regulatory activities, with nTregs preferentially involved in preserving self-tolerance and functionally active iTregs present at inflammatory sites $[49,50]$. Unlike effector $\mathrm{T}$ cells, Tregs demonstrate a low level of mTOR activity throughout their lives [51]. In fact, their development occurs in conditions that promote minimal mTOR activity, such as low affinity or brief TCR binding and stimulation $[52,53]$.

Emerging data suggest that the number and function of Tregs in humans are maintained even in senescence [54], supporting the relevance of those peripherally developed iTregs. Recent evidence emphasizes the functional plasticity of $\mathrm{T}$ cells. In this context, iTregs can be generated from Th1, Th2, and Th17 effector cells as well as from naive cells [55,56]. Our group has demonstrated that human primary naive $\mathrm{CD} 4^{+} \mathrm{T}$ cells differentiate into Tregs only after multiple rounds of cell division and re-configuration of the PI3K/ AKT/ mTOR pathway [2,3]. This is similar to the "two-step differentiation model" proposed by Guo et al. [57] according to which the initial TCR-dependent conditioning step induces the expression of CD25 and "primes" the cell to efficiently respond to the second "cytokine-dependent" phase that results in the acquisition of the Treg phenotype.

Several signals have been described to govern the generation, function and stability of Treg cells. Smad3 plays a critical role in promoting regulatory $\mathrm{T}$ cell differentiation. TGF- $\beta$ signaling activates Smad3 which, along with mTOR-induced NF-AT, contributes to the induction of Foxp3 by promoting acetylation of the FOXP3 enhancer. mTOR plays simultaneous roles of inducing specific effector $\mathrm{T}$ cell differentiation by enhancing STAT activation and inhibiting Smad3 phosphorylation. Delgoffe et al. [36] suggested that in the presence of active MTOR, TCR engaged $\mathrm{T}$ cells display normal IL-2 production and differentiate towards Th1, Th2 and Th17. However, the absence of mTOR leads to induction of functionally stable Treg cells. The authors propose that in the absence of mTOR, mTORC2-mediated activation of AKT is absent, resulting in hyper phosphorylation of Smad3. Our laboratory has shown that the expression of AKT protein is substantially higher in the Treg subpopulation when compared to conventional $\mathrm{T}$ cells, likely due to the increased stability of non-active AKT. Moreover, this low-active AKT in Tregs coimmunoprecipitates with Smad3, suggesting a new level of crosstalk between the TFG- $\beta$ and TCR pathways in iTregs (data not published).

Critical among the signals that guide the development and function of Tregs are also those mediated by IL-2R, as an increase in this pathway has been associated with an accumulation of Treg cells in vivo [58]. Among the signals activated via IL-2R, the phosphorylation of the transcription factor STAT5 appears to play a key role in the generation and expansion of Treg cells. IL-2 also initiates signaling through PI3K. Tregs express high levels of PTEN, the main negative regulator of PI3K suggesting a restrained activation of PI3K signaling. Huynh et al. [59] demonstrated that PTEN-mediated control of PI3K activity is critical for the stability of mouse Tregs. Using a PTEN-deficient mouse model, specifically in the Foxp $3^{+}$population, they found that decreased control of PI3K activity led to the accumulation of transient Foxp $3^{+} \mathrm{CD} 25$ cells and subsequent loss of expression of Foxp3.

Other mTOR-dependent pathways may negatively regulate Foxp3 expression. The FOXO transcription factors directly bind to the Foxp3 promoter, and they are inactivated by mTORC2dependent, AKT-mediated phosphorylation. Moreover, the expression of the constitutively active form of AKT in Treg cells has been associated with a diminished Treg cell gene signature, including reduced expression of Foxp3 and IL2ra (IL2R $\alpha$ subunit CD25) [60]. The fact that an increase in Tregs was observed following deletion of mTOR but not in the Rheb and Rictor T cell conditional knockouts suggests that loss of both of these pathways is necessary to enhance the generation of Tregs [43]. In the same context, the rapamycin-induced blockage of mTOR signaling also generates Tregs through induction of Foxp3 expression regardless of the presence of effector $\mathrm{T}$ cell skewing cytokines and strong TCR engagement.

The above-mentioned balance of the feedback loops between mTORC1 and mTORC2 may impact the effects of rapamycinbased therapy, which was initially described as a selective allosteric inhibitor of mTORC1. Blocking the mTORC1-dependent S6K activation stops the negative feedback loop over PI3K, which leads to hyper activation of AKT. Therefore, the single targeting of mTOR has to be carefully addressed in a cell type-specific, time and concentration dependent-manner.

\section{mTOR as a mediator of the metabolism of proliferating T cells}

In contrast to the rather well characterized intracellular signaling cascades, the role of cytokine-induced metabolic changes in $\mathrm{T}$ cell fate and function have been recognized only recently [61]. mTOR is known to play a key role in integrating signals that respond to nutrient availability and growth factors from the extracellular environment and to adjust the cell's metabolism accordingly.

In the quiescent metabolic state of naive $\mathrm{T}$ cells, the low activity of mTOR correlates with the mitochondrial fatty acid oxidation as the primary energy source of the cell [62]. Upon 
effective antigen recognition, the clonal expansion of antigenprimed $\mathrm{T}$ cells demands the sudden increase in aerobic glycolysis to support their new energetic and anabolic demands. This preferential metabolic circuitry in functionally active $\mathrm{T}$ cells is similar to the aerobic glycolysis of cancer cells, a phenomenon known as the Warburg effect. Recent studies have identified specific signaling events and transcription factors involved in the coordination of cell proliferation and metabolism in activated $\mathrm{T}$ cells that might dictate the outcome of adaptive immune responses. Likewise, there is a growing body of evidence coupling the plasticity of cell metabolism and bioenergetic pathways with T cell fate lineage decisions. PI3K/ mTOR signaling plays a central role in the metabolic regulation of effector $\mathrm{T}$ cell activation and differentiation.

The activation of antigen-engaged naive $\mathrm{T}$ cells requires a rapid mTORC1-dependent readjustment of the metabolic machinery towards glycolysis [63]. The glycolytic switch in antigen-experienced cells is mTORC2-dependent [64]. These findings depict a model of evolving mTOR-complex prevalence in the regulation of effector $\mathrm{T}$ cell responses and emphasize the key role of mTOR activity in the metabolic reprogramming of $\mathrm{T}$ cells. The integration of $\mathrm{T}$ cell metabolism and function is especially relevant for the maintenance of the immune homeostasis between effector and regulatory $\mathrm{T}$ cell function. While pro- inflammatory effector Th1, Th2 and Th17 activities rely mostly on glycolysis to meet the cell energy demands, both natural and iTreg cells and $\mathrm{CD}^{+}$memory $\mathrm{T}$ cells do not primarily use glycolysis for energy generation and instead rely on fatty acid metabolism [65]. Compared to effector T cells, memory T cells have enhanced mitochondrial spare respiratory capacity and greater mitochondrial mass than naive T cells, which provides the extra energy necessary to promote survival [65-67]. Targeting cell metabolism is envisioned as a novel therapeutic strategy in cancer but is also emerging as an attractive immunotherapeutic approach [Figure 3].

\section{Perspectives of pharmacological targeting of mTor signaling pathway on treg development}

The physiologic relevance and potential involvement of the mTOR pathway as the facilitator of T cell lineage commitment in several pathological conditions have attracted major interest as a pharmacological target. Inhibition or deficiency in this pathway may lead to acquisition or increase of suppressor Treg function, and attracted interest as pharmacological target in autoimmune disease, transplantation and graft versus host disease (GVHD).

Rapamycin promotes de novo expression of Foxp3 in naive T cells, and it has demonstrable beneficial effects on the survival and proliferation of Tregs in vivo and in vitro [6,7]. As a result,

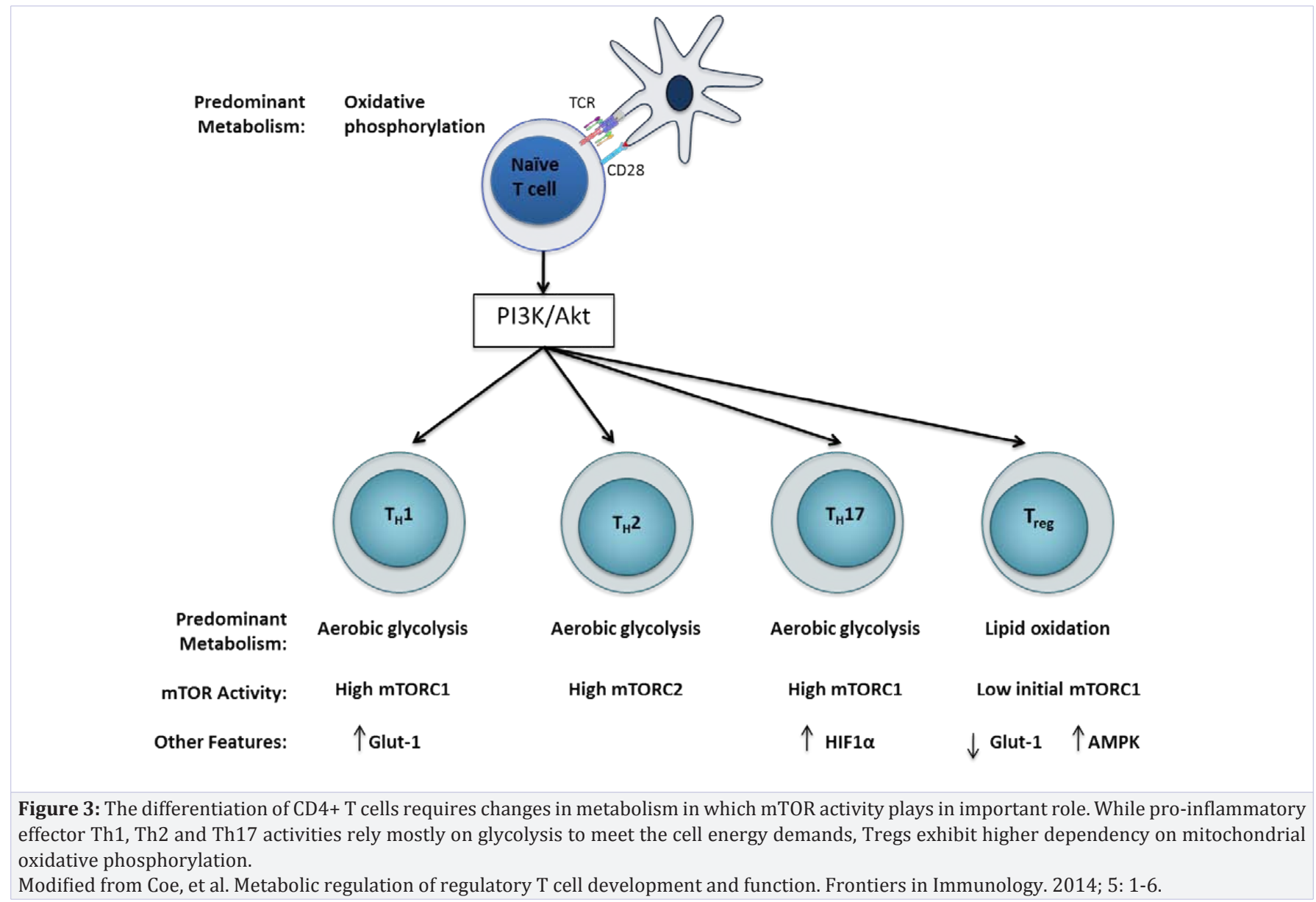

Citation: Coquillard C, Vilchez V, Marti F, Gedaly R (2015) mTOR Signaling in Regulatory T Cell Differentiation and Expansion. SOJ Immunol 3(1): 1-10. DOI: http://dx.doi.org/10.15226/soji/3/1/00122 
rapamycin inhibits effector $\mathrm{T}$ cell proliferation but promotes Treg accumulation. As previously mentioned, the preferential inhibition of mTORC1 by rapamycin may lead to non-desirable effects such as losing the inhibitory effect of S6K over PI3Kinduced AKT activation. Alternative therapeutic strategies have been proposed that combine mTOR inhibition and upstream PI3K/AKT inactivation in cancer [68].

One of the limitations in developing Treg-based therapies is the low frequency of circulating Tregs, especially antigen-specific Tregs, and the unfavorable ex vivo expansion properties. However, recent technical advances in ex vivo expansion regimens of nTregs or generation of iTregs have made it feasible to reconsider Treg therapies [69-71]. Ex vivo conversion of $\mathrm{CD} 4^{+} \mathrm{CD} 25^{-}$naive T cells into iTregs with suppressor function represents an alternative strategy to ex vivo nTreg isolation and expansion. Our group [4] and others have shown that Treg culture media with relative low dose of IL-2 in the presence of rapamycin resulted in a steady and consistent growth of Treg cells essentially free of contaminating non-Tregs. The high degree of plasticity and insufficient number of antigen-specific Tregs that can be obtained with ex-vivo expansion still represents major challenges associated with the infusion of human Tregs in clinical practice.

Several phase I trials have been conducted to assess their effect on GVHD following hematopoietic stem cell transplantation (HSCT) and in type I diabetes [72]. The first human trial employing adoptive transfer of nTregs to suppress GVHD was reported by Edinger et al. [73] treating five HSCT recipients with fresh, bead-purified donor nTregs. The authors reported no infusional toxicity, increase in infection rate or GVHD. A similar study using purified nTregs was performed in 2008 by Di Ianni et al. [74] to evaluate the effect of these cells on GVHD prevention in 28 patients. nTregs were infused into patients three days prior to $\mathrm{CD} 34^{+}$cells supplemented with frozen/thawed mature donor $\mathrm{T}$ cells in the absence of any post-transplant immunosuppression. The authors showed that adoptive transfer of Tregs prevented GVHD, promoted lymphoid reconstitution and enhanced immunity to opportunistic infections. Trzonkowski et al. [75] used sort-purified nTregs (CD4 $\left.4^{+} 5^{+} 127^{-}\right)$expanded in vitro to treat two patients with GVHD with overall positive outcomes. More recently, Brunstein et al. [76] established a method of $\mathrm{CD}^{+}{ }^{+} \mathrm{CD} 25^{+} \mathrm{FoxP} 3^{+}$Treg enrichment from cryopreserved umbilical cord blood (UCB), and evaluated the safety profile of these cells in 23 patients. The authors reported no infusional toxicities with a reduced incidence of acute GVHD when compared to control subjects. These initial clinical trials evaluating the safety and efficacy of Tregs in treating GVHD demonstrate promising safety and potentially efficacy profiles and increase the interest to consider wider applications of Treg-based therapy in other diseases including autoimmunity and solid organ transplantation $[77,78]$.

Beta-cell specific auto reactive T cells can be found in patients with Type I Diabetes (T1D) and in healthy controls, and are usually controlled by a network of regulatory mechanisms including Treg cells. Increasing the number of Tregs by adoptive transfer can be used to prevent and treat even established T1D [79]. Putnam et al. [80] isolated Tregs from recent-onset T1D patients and healthy control subjects by fluorescence-activated cell sorting and compared their capacity to expand in vitro. The authors found that expansion of $\mathrm{CD} 4{ }^{+} \mathrm{CD} 127^{\mathrm{lo} /}$ cells required the addition of rapamycin to maintain lineage purity. In contrast, expansion of $\mathrm{CD} 4^{+} \mathrm{CD} 127^{\mathrm{lo} /} \mathrm{CD} 25^{+} \mathrm{T}$-cells, resulted in high yield, functional Tregs that maintained higher Foxp3 expression in the absence of rapamycin. Tregs from T1D patients and control subjects expanded similarly and were equally capable of suppressing T-cell proliferation. Pre-clinical data support that low dose IL-2 drives tolerance via Treg activation and that the IL-2/IL-2R axis is impaired in T1D [81]. Rapamycin/ IL-2 combination treatment of NOD mice effectively treats autoimmune diabetes [82]. Based on these findings, Long et al. [83] performed a phase 1 clinical trial to test the safety and immunologic effects of rapamycin/ IL-2 combination therapy in T1D patients. Nine subjects were treated with rapamycin for three months and IL-2 for one month. The authors found that Treg cells transiently increased within the first month of therapy, although the response of these cells to IL-2 (as measured by STAT5 phosphorylation) improved and persisted after treatment. Notably, clinical and metabolic data demonstrated a transient worsening in all subjects.

In solid organ transplantation, a growing body of evidence recognizes the balance between graft-reactive effector cells and graft-protective suppressor Tregs as the ultimate determinant of long-term allograft survival, in which mTOR pharmacological inhibition may play a role [84]. Higher levels of circulating Tregs have been linked to better outcomes and higher percentages of Tregs within $\mathrm{T}$ cell infiltrates in transplant biopsies and have been also correlated with better function of transplanted organs [85,86]. In 2013, Yamashita et al. [87] reported the administration of in vitro generated iTregs to 10 patients undergoing liver transplantation. Administration of these cells was safe and appeared to facilitate early weaning of immunesuppression in half of the patients. These findings are promising but preliminary, and long-term outcomes in large cohorts should be evaluated to determine the real benefit of Treg-based therapy in solid organ transplantation. Funded by the European Commission's Seventh Framework, the ONE Study, currently underway, is a Phase I/ IIa clinical trial designed to test the safety and practicality of seven different regulatory cell populations in living donor kidney transplantation [88].

A deeper understanding of the activity of mTOR in regulatory $\mathrm{T}$ cells will help elucidate the uniqueness of this signaling and will open novel doors toward therapeutic manipulation of Tregs. Treg-based cellular therapy has not caused any infusional toxicity and it has established a limited safety record with regard to risk of infection, relapse or early mortality. However, efficacy data is still currently lacking.

In summary, the mTOR signaling pathway is critical in $\mathrm{T}$ cell fate decisions that promote a reduction of effector $\mathrm{T}$ cells and differentiation and expansion of regulatory T cells. A better understanding of the complexities of the PI3K/ AKT/ mTOR pathway in the regulation of $\mathrm{T}$ cells will be essential in designing novel therapeutic strategies for future applications in cancer, immunology/ allergy and clinical transplantation. 


\section{References}

1. Shaw R, Cantley LC. Ras PI, (3)K and mTOR signaling controls tumour cell growth. Nature. 2006; 441(7092): 424-430.

2. Zoncu R, Efeyan A, Sabatini DM. mTOR: from growth signal integration to cancer, diabetes and ageing. Nat Rev Mol Cell Biol. 2011; 12(1): 21 35. doi: $10.1038 / \mathrm{nrm} 3025$.

3. Kim DH, Sarbassov DD, Ali SM, King JE, Latek RR, Erdjument-Bromage $\mathrm{H}$, et al. mTOR interacts with raptor to form a nutrient-sensitive complex that signals to the cell growth machinery. Cell. 2002; 110(2): 163-175.

4. Ellis GI, Reneer MC, Vélez-Ortega AC, McCool A, Martí F. Generation of induced regulatory $\mathrm{T}$ cells from primary human naive and memory $\mathrm{T}$ cells. J Vis Exp. 2012; (62): 3738. doi: 10.3791/3738.

5. Reneer MC, Estes DJ, Vélez-Ortega AC, Norris A, Mayer M, Marti F. Peripherally induced human regulatory $\mathrm{T}$ cells uncouple Kv1.3 activation from TCR-associated signaling. Eur Immunol. 2011; 41(11):3170-3175. doi: 10.1002/eji.201141492.

6. McMahon G, Weir MR, Li XC, Mandelbrot DA. The Evolving Role of mTOR inhibition in Transplantation Tolerance. J Am SocNephrol 2011; 22(3): 408-415. doi: 10.1681/ASN.2010040351.

7. Battaglia M, Stabilini A, Roncarolo MG. Rapamycin selectively expands $\mathrm{CD}^{+}{ }^{\mathrm{CD}} 25^{+} \mathrm{FoxP}^{+}$regulatory $\mathrm{T}$ cells. Blood. 2005; 105(12): $4743-$ 4748.

8. Dobashi Y, Watanabe Y, Miwa C, Suzuki S, Koyama S. Mammalian target of rapamycin: a central node of complex signaling cascades. Int J Clin Exp Pathol. 2011; 4(5): 476-495.

9. Ma XM, Blenis J. Molecular mechanisms of mTOR-mediated translational control. Nat Rev Mol Cell Biol. 2009; 10(5): 307-318. doi: $10.1038 / \mathrm{nrm} 2672$

10. Sarbassov DD, Ali SM, Kim DH, Guertin DA, Latek RR, ErdjumentBromage $\mathrm{H}$, et al. Rictor, a novel binding partner of mTOR, defines a rapamycin-insensitive and raptor-independent pathway that regulates the cytoskeleton. Curr Biol. 2004; 14(14): 1296-1302.

11.Vander Haar E, Lee SI, Bandhakavi S, Griffin TJ, Kim DH. Insulin signalling to mTOR mediated by the Akt/PKB substrate PRAS40. Nat Cell Biol. 2007; 9(3): 316-323.

12. Lu M, Wang J, Ives HE, Pearce D. mSIN1 protein mediates SGK1 protein interaction with mTORC2 protein complex and is required for selective activation of the epithelial sodium channel. J Biol Chem. 2011; 286(35): 30647-30654. doi: 10.1074/jbc.M111.257592.

13. Pearce LR, Sommer EM, Sakamoto K, Wullschleger S, Alessi DR. Protor-1 is required for efficient mTORC2-mediated activation of SGK1 in the kidney. Biochem J. 2011; 436(1): 169-179. doi: 10.1042/ BJ20102103.

14. Laplante M, Sabatini DM. mTOR signaling at a glance. J Cell Sci. 2009; 122(10): 3589-3594. doi: 10.1242/jcs.051011.

15. Shimobayashi M, Hall MN. Making new contacts: the mTOR network in metabolism and signalling crosstalk. Nat Rev Mol Cell Biol. 2014; 15(3): 155-162. doi: 10.1038/nrm3757.

16. Sarbassov DD, Ali SM, Sengupta S, Sheen JH, Hsu PP, Bagley AF, et al. Prolonged rapamycin treatment inhibits mTORC2 assembly and Akt/ PKB. Mol Cell. 2006; 22(2): 159-168.

17. Bracho-Valdes I, Moreno-Alvarez P, Valencia-Martines I, RoblesMolina E, Chavez-Vargas L, Vazquez-Prado J. mTORC1- and mTORC2 interacting proteins keep their multifunctional partners focused. Life.

\section{1; 63(10): 896-914. doi: 10.1002/iub.558.}

18. Vanhaesebroeck B, Guillermet-Guibert J, Graupera M, Bilanges B. The emerging mechanisms of isoform-specific PI3K signaling. Nat Rev Mol Cell Biol. 2010; 11(5): 329-341. doi: 10.1038/nrm2882.

19. Long X, Lin Y, Ortiz-Vega S, Yonezawa K, Avruch J. Rheb binds and regulates the mTOR kinase. Curr Biol. 2005; 15(8): 702-713.

20. Tee AR, Manning BD, Roux PP, Cantley LC, Blenis J. Tuberous Sclerosis Complex Gene Products, Tuberin and Hamartin, Control mTOR Signaling by Acting as a GTPase-Activating Protein Complex toward Rheb. Current Biology. 2003; 13(15): 1259-1268.

21. Cai SL, Tee AR, Short JD, Bergeron JM, Kim J, Shen K, et al. Activity of TSC2 is inhibited by AKT-mediated phosphorylation and membrane partitioning. J Cell Biol. 2006; 173(2): 279-289.

22. Carracedo A, Pandolfi PP. The PTEN-PI3K pathway: of feedbacks and cross-talks. Oncogene. 2008; 27(41): 5527-5541. doi: 10.1038/onc.

23. Carriere A, Romeo Y, Acosta-Jaquez HA, Moreau J, Bonneil E, Thibault $\mathrm{P}$, et al. ERK1/2 phosphorylate Raptor to promote Ras-dependent activation of mTOR complex 1 (mTORC1). J Biol Chem. 2011; 286(1): 567-577. doi: 10.1074/jbc.M110.159046.

24.Dan HC, Baldwin AS. Differential involvement of IkappaB kinases alpha and beta in cytokine- and insulin-induced mammalian target of rapamycin activation determined by Akt. J Immunol. 2008; 180(11): 7582-7589.

25. Guertin DA, Sabatini DM. Defining the role of mTOR in cancer. Cancer Cell. 2007; 12(1): 9-22

26. Gwinn DM, Shackelford DB, Egan DF, Mihaylova MM, Mery A Vasquez DS, et al. AMPK phosphorylation of raptor mediates a metabolic checkpoint. Mol Cell. 2008; 30(2): 214-226. doi: 10.1016/j. molcel.2008.03.003.

27. Chauvin C, Koka V, Nouschi A, Mieulet V, Hoareau-Aveilla C, Dreazen A, et al. Ribosomal protein S6 kinase activity controls the ribosome biogenesis transcriptional program. Oncogene. 2014; 33(4): 474-483. doi: 10.1038/onc.2012.606.

28. Harrington LS, Findlay GM, Gray A, Tolkacheva T, Wigfield S, Rebholz $\mathrm{H}$, et al. The TSC1-2 tumor suppressor controls insulin-PI3K signaling via regulation of IRS proteins. The Journal of Cell Biology. 2004; 166(2): 213-223.

29. Zhang H, Bajraszewski N, Wu E, Wang H, Moseman AP, Dabora SL, et al. PDGFRs are critical for PI3K/Akt activation and negatively regulated by mTOR. J Clin Invest. 2007; 117(3):730-738.

30.Zinzalla V, Stracka D, Oppliger W, Hall MN. Activation of mTORC2 by association with the ribosome. Cell. 2011; 144(5): 757-768. doi: 10.1016/j.cell.2011.02.014

31.Appenzeller-Herzog C, Hall MN. Bidirectional crosstalk between endoplasmic reticulum stress and mTOR signaling. Trends Cell Biol. 2012; 22(5): 274-282. doi: 10.1016/j.tcb.2012.02.006.

32. Efeyan A, Sabatini DM. mTOR and cancer: many loops in one pathway. Current Opinion in Cell Biology. 2010; 22: 169-176. doi: 10.1016/j. ceb.2009.10.007.

33. Laplante M, Sabatini DM. mTOR signaling in growth control and disease. Cell. 2012,13; 149(2): 274-93. doi: 10.1016/j.cell.2012.03.017.

34.Xie J, Proud CG. Crosstalk between mTOR complexes. Nat Cell Biol. 2013; 15(11):1263-1265. doi: 10.1038/ncb2877.

35. Sprent J, Surh CD. Normal T cell homeostasis: the conversion of naive cells into memory-phenotype cells. Nat Immunol. 2011; 131(6): 478- 
484.

36. Delgoffe GM, Kole TP, Zheng Y, Zarek PE, Matthews KL, Xiao B, et al. The mTOR kinase differentially regulates effector and regulatory $\mathrm{T}$ cell lineage commitment. Immunity. 2009; 30(6): 832-844. doi: 10.1016/j.immuni.2009.04.014.

37. O'Brien TF, Gorentla BK, Xie D, Srivatsan S, McLeod IX, He YW, et al. Regulation of T-cell survival and mitochondrial homeostasis by TSC1. Eur J Immunol. 2011; 41(11): 3361-3370. doi: 10.1002/ eji.201141411.

38.Wu Q, Liu Y, Chen C, Ikenoue T, Qiao Y, Li CS, et al. The tuberous sclerosis complex-mammalian target of rapamycin pathway maintains the quiescence and survival of naive T cells. J Immunol. 2011; 187(3): 1106-1112. doi: 10.4049/jimmunol.1003968.

39. Schwartz RH. T cell anergy. Annu Rev Immunol. 2003; 21: 305-334.

40. Koch U, Radtke F. Mechanisms of $\mathrm{T}$ cell development and transformation. Annu Rev Cell Dev Biol. 2011; 27: 539-562. doi: 10.1146/annurev-cellbio-092910-154008.

41. Waickman AT, Powell JD. mTOR, metabolism, and the regulation of T-cell differentiation and function. Immunol Rev. 2012; 249(1): 4358. doi: 10.1111/j.1600-065X.2012.01152.x.

42.Zhu J, Yamane H, Paul WE. Differentiation of effector CD4 T cell populations $\left({ }^{*}\right)$. Annu Rev Immunol. 2010; 28: 445-89. doi: 10.1146/ annurev-immunol-030409-101212.

43. Delgoffe GM, Pollizzi KN, Waickman AT, Heikamp E, Meyers DJ, Horton MR, et al. The kinase mTOR regulates the differentiation of helper T cells through the selective activation of signaling by mTORC1 and mTORC2. Nat Immunol. 2011; 12(4): 295-303. doi: 10.1038/ni.2005.

44. Lee K, Gudapati P, Dragovic S, Spencer C, Joyce S, Killeen N, et al. Mammalian target of rapamycin protein complex 2 regulates differentiation of Th1 and Th2 cell subsets via distinct signaling pathways. Immunity. 2010; 32(6): 743-53. doi: 10.1016/j. immuni.2010.06.002.

45. Heikamp EB, Patel CH, Collins S, Waickman A, Oh MH, Sun IH, et al. The AGC kinase SGK1 regulates TH1 and TH2 differentiation downstream of the mTORC2 complex. Nat Immunol. 2014; 15(5): 457-64. doi: $10.1038 /$ ni.2867

46. Vignali DA, Collison LW, Workman CJ. How regulatory T cells work. Nat Rev Immunol. 2008; 8(7): 523-32. doi: 10.1038/nri2343.

47. Lastovicka J. The Phenotypic Markers of CD4+CD25+ T Regulatory Lymphocytes. Research in Immunology: An International Journal. 2013; 2013.

48. Horwitz DA, Zheng SG, Gray JD. Natural and TGF-beta-induced Foxp3(+)CD4(+) CD25(+) regulatory $\mathrm{T}$ cells are not mirror images of each other. Trends Immunol. 2008; 29(9): 429-35. doi: 10.1016/j. it.2008.06.005.

49. Bluestone JA, Abbas AK. Natural versus adaptive regulatory T cells. Nat Rev Immunol. 2003; 3(3):253-7.

50. Reneer MC, Marti F. The balancing act of AKT in T cells. Front Biol. 2013; 8(2): 160-74.

51.Zeiser R, Leveson-Gower DB, Zambricki EA, Kambham N, Beilhack A, Loh J, et al. Differential impact of mammalian target of rapamycin inhibition on CD4+CD25+Foxp3+ regulatory $\mathrm{T}$ cells compared with conventional CD4+ T cells. Blood. 2008; 111(1): 453-62.

52. Gottschalk RA, Corse E, Allison JP. TCR ligand density and affinity determine peripheral induction of Foxp3 in vivo. J Exp Med. 2010;

\section{7(8): 1701-11. doi: 10.1084/jem.20091999. .}

53. Katzman SD, O'Gorman WE, Villarino AV, Gallo E, Friedman RS, Krummel MF, et al. Duration of antigen receptor signaling determines T-cell tolerance or activation. Proc Natl Acad Sci. 2010; 107(42): 18085-90. doi: 10.1073/pnas.1010560107.

54. Jagger A, Shimojima Y, Goronzy JJ, Weyand CM. Regulatory T cells and the Immune Aging Process: A mini-review. Gerontology 2014; 60(2):130-7. doi: 10.1159/000355303.

55. Hori S. Developmetal plasticity of Foxp3+ regulatory T cells. Curr Opin Immunol. 2010; 22(5): 575-82. doi: 10.1016/j.coi.2010.08.004.

56.Zhou L, Lopes JE, Chong MM, Ivanov II, Min R, Victora GD, et al. TGFbeta-induced Foxp3 inhibits Th17 cell differentiation by antagonizing ROR gamma function. Nature. 2008; 453(7192): 236-40.

57.Guo Z, Khattar M, Schroder PM, Miyahara Y, Wang G, He X, et al. A dynamic dual role of IL-2 signaling in the two-step differentiation process of adaptive regulatory T cells. J Immunol. 2013; 190(7): 3153-62. doi: 10.4049/jimmunol.1200751.

58. Webster KE, Walters S, Kohler RE, Mrkvan T, Boyman O, Surh CD, et al. In vivo expansion of T reg cells with IL-2-mAb complexes: induction of resistance to EAE and long-term acceptance of islet allografts without immunosuppression. J Exp Med. 2009; 206(4): 751-60. doi: 10.1084/ jem.20082824.

59. Huynh A, DuPage M, Priyadharshini B, Sage PT, Quiros J, Borges CM, et al. Control of PI(3) kinase in Treg cels maintains homeostasis and lineage stability. Nat Immunol. 2015; 16(2): 188-96. doi: 10.1038/ ni.3077.

60. Haxhinasto S, Mathis D, Benoist C. The AKT-mTOR axis regulates de novo differentiation of CD4+Foxp3+ cells. J Exp Med. 2008; 205(3): 565-74. doi: 10.1084/jem.20071477.

61.Wang R, Green DR. Metabolic checkpoints in activated T cells. Nat Immunol. 2012; 13(10): 907-15. doi: 10.1038/ni.2386.

62. Wang R, Dillon CP, Shi LZ, Milasta S, Carter R, Finkelstein D, et al. The transcription factor Myc controls metabolic reprogramming upon T lymphocyte activation. Immunity. 2011; 35(6): 871-82. doi: 10.1016/j.immuni.2011.09.021.

63. Pearce EL, Poffenberger MC, Chang $\mathrm{CH}$, Jones RG. Fueling immunity: insights into metabolism and lymphocyte function. Science. 2013; 342(6155): 1242454. doi: 10.1126/science.1242454.

64.Gubser PM, Bantug GR, Razik L, Fischer M, Dimeloe S, Hoenger G, Durovic B, et al. Rapid effector function of memory CD8+ T cells requires an immediate-early glycolytic switch. Nat Immunol. 2013; 14(10): 1064-72. doi: 10.1038/ni.2687.

65. Pollizzi KN, Powell JD. Integrating canonical and metabolic signalling programmes in the regulation of T cell responses. Nat Rev Immunol. 2014 ;14(7): 435-46. doi: 10.1038/nri3701.

66. Van der Windt GJ, O’Sullivan D, Everts B, Huang SC, Buck MD, Curtis JD, et al. CD8 memory T cells have a bioenergetic advantage that underlies their rapid recall ability. Proc Natl Acad Sci U S A. 2013;110(35): 14336-41. doi: 10.1073/pnas.1221740110.

67. Pearce EL, Walsh MC, Cejas PJ, Harms GM, Shen H, Wang LS, et al. Enhancing CD8 T-cell memory by modulating fatty acid metabolism. Nature. 2009; 460(7251): 103-7. doi: 10.1038/nature08097.

68. Maira SM, Stauffer F, Brueggen J, Furet P, Schnell C, Fritsch C, et al. Identification and characterization of NVP-BEZ235, a new orally available dual phosphatidylinositol 3-kinase/mammalian target of rapamycin inhibitor with potent in vivo antitumor activity. Mol Cancer 
Ther. 2008; 7(7): 1851-63. doi: 10.1158/1535-7163.MCT-08-0017.

69. Levings MK, Sangregorio R, Roncarolo MG. Human CD25(+)CD4(+) T regulatory cells suppress naive and memory $\mathrm{T}$ cell proliferation and can be expanded in vitro without loss of function. J Exp Med. 2001; 193(11): 1295-302.

70. Earle KE, Tang Q, Zhou X, Liu W, Zhu S, Bonyhadi ML, et al. In vitro expanded human CD4+CD25+ regulatory $\mathrm{T}$ cells suppress effector $\mathrm{T}$ cell proliferation. Clin Immunol. 2005; 115(1): 3-9.

71. Tang Q, Henriksen KJ, Bi M, Finger EB, Szot G, Ye J, et al. In vitroexpanded antigen-specific regulatory $\mathrm{T}$ cells suppress autoimmune diabetes. J Exp Med. 2004; 199(11): 1455-65.

72. Hippen KL, Riley JL, June CH, Blazar BR. Clinical perspectives for regulatory T cells in transplantation tolerance. SeminImmunol. 2011; 23(6): 462-8. doi: 10.1016/j.smim.2011.07.008.

73. Hoffmann P, Boeld TJ, Eder R, Albrecht J, Doser K, Piseshka B, et al. Isolation of CD4+CD25+ regulatory T cells for clinical trials. Biol Blood Marrow Transplant. 2006; 12(3): 267-74.

74. Di Ianni M, Falzetti F, Carotti A, Terenzi A, Castellino F, Bonifacio E, et al. Tregs prevent GVHD and promote immune reconstitution in HLAhaploidentical transplantation. Blood. 2011; 117(14): 3921-3928. doi: 10.1182/blood-2010-10-311894.

75. Trzonkowski P, Bieniaszewska M, Juscinska J, Dobyszuk A, Krzystyniak A, Marek N, et al. First-in-man clinical results of the treatment of patients with graft versus host disease with human ex vivo expanded CD4+CD25+CD127- T regulatory cells. Clin Immunol. 2009; 133(1): 22-26. doi: 10.1016/j.clim.2009.06.001.

76. Brunstein CG, Miller JS, Cao Q, McKenna DH, Hippen KL, Curtsinger J, et al. Infusion of ex vivo expanded T regulatory cells in adults transplanted with umbilical cord blood: safety profile and detection kinetics. Blood. 2011; 117(3): 1061-1070. doi: 10.1182/blood-2010-07-293795.

77. Tang 0. T-reg: The Ups and Downs. ESOT+AST Meeting 2014 Personalized Transplantation. Madrid (Spain).

78. Veerapathran A, Pidala J, Beato F, Yu XZ, Anasetti C. Ex vivo expansion of human Tregs specific for alloantigens presented directly or indirectly. Blood. 2011; 118(20): 5671-5680. doi: 10.1182/ blood-2011-02-337097.
79. Jaeckel E, Mpofu N, Saal N, Manns MP. Role of regulatory T cells for the treatment of type 1 diabetes mellitus. Horm Metab Res. 2008; 40(2): 126-136. doi: 10.1055/s-2008-1042427.

80. Putnam AL, BruskoTM, Lee MR, Liu W, Szot GL, Ghosh T, et al. Expansion of Human Regulatory T-Cells From Patients With Type 1 Diabetes. Diabetes. 2009; 58(3): 652-662. doi: 10.2337/db08-1168.

81. Bayer AL, Pugliese A. The IL-2/IL-2R system: from basic science to therapeutic applications to enhance immune regulation. Immunol Res. 2013; 57(1-3): 197-209. doi: 10.1007/s12026-013-8452-5.

82. Long SA, Buckner JH, Greenbaum CJ. IL-2 therapy in type 1 diabetes: "Trials" and tribulations. Clinical Immunology. 2013; 149(3): 324331. doi: 10.1016/j.clim.2013.02.005.

83. Long SA, Rieck M, Sanda S, Bollyky JB, Samuels PL, Goland R, et al. Rapamycin/IL-2 combination therapy in patients with type 1 diabetes augments Tregs yet transiently impairs beta-cell function. Diabetes. 2012; 61(9): 2340-2348. doi: 10.2337/db12-0049.

84. Tang Q, Bluestone JA, Kang SM. CD4(+)Foxp3(+) regulatory T cell therapy in transplantation. J Mol Cell Biol 2011; 4(1): 11-21. doi: 10.1093/jmcb/mjr047.

85. Demirkiran A, Kok A, Kwekkeboom J, Kusters JG, Metselaar HJ, Tilanus HW, et al. Low circulatory regulatory T-cell levels after acute rejection in liver transplantation. Liver Transpl 2006; 12(2): 277-284.

86. Bestard O, Cruzado JM, Rama I, Torras J, Goma M, Seron D, et al. Presence of Foxp3+ regulatory T cells predicts outcome of subclinical rejection of renal allografts. J Am Soc Nephrol 2008; 19(10): 2020 2026. doi: 10.1681/ASN.2007111174.

87. Yamashita K, Zaitsu M, Nagatsu A, et al. A clinical trial of regulatory T cell-based immunotherapy for tolerance induction in living donor liver transplantation. Vienna: European Society for Organ Transplantation, 2013.

88. Geissler EK. The ONE study compares cell therapy products in organ transplantation: Introduction to a review series on suppressive monocyte-derived cells. Transplant Res 2012; 1:11-13. 